\title{
Evaluation of diastolic function by three-dimensional volume tracking of the mitral annulus with cardiovascular magnetic resonance: comparison with tissue Doppler imaging
}

Vincent $\mathrm{Wu}^{1}$, Janice Y Chyou ${ }^{2}$, Sohae Chung ${ }^{1}$, Sharath Bhagavatula ${ }^{1}$ and Leon Axel ${ }^{1,2^{*}}$

\begin{abstract}
Background: Measurement of mitral annulus (MA) dynamics is an important component of the evaluation of left ventricular (LV) diastolic function; MA velocities are commonly measured using tissue Doppler imaging (TDI). This study aimed to examine the clinical potential of a semi-automated cardiovascular magnetic resonance (CMR) technique for quantifying global LV diastolic function, using 3D volume tracking of the MA with conventional cine-CMR images.

Methods: 124 consecutive patients with normal ejection fraction underwent both clinically indicated transthoracic echocardiography (TTE) and CMR within 2 months. Interpolated 3D reconstruction of the MA over time was performed with semi-automated atrioventricular junction (AVJ) tracking in long-axis cine-CMR images, producing an MA sweep volume over the cardiac cycle. CMR-based diastolic function was evaluated, using the following parameters: peak volume sweep rates in early diastole $\left(P S R_{E}\right)$ and atrial systole $\left(P S R_{A}\right), P S R_{E} / P S R_{A}$ ratio, deceleration time of sweep volume (DT $\mathrm{SV}$ ), and 50\% diastolic sweep volume recovery time (DSVRT ${ }_{50}$ ); these were compared with TTE diastolic measurements.
\end{abstract}

Results: Patients with TTE-based diastolic dysfunction $(n=62)$ showed significantly different normalized MA sweep volume profiles compared to those with TTE-based normal diastolic function $(n=62)$, including a lower $\operatorname{PSR}_{E}\left(5.25 \pm 1.38 \mathrm{~s}^{-1}\right.$ vs. $\left.7.72 \pm 1.7 \mathrm{~s}^{-1}\right)$, a higher PSR $\left(6.56 \pm 1.99 \mathrm{~s}^{-1}\right.$ vs. $\left.4.67 \pm 1.38 \mathrm{~s}^{-1}\right)$, a lower PSR $/$ PSR ratio $(0.9 \pm 0.44$ vs. $1.82 \pm 0.69)$, a longer DT Sv $(144 \pm 55 \mathrm{~ms}$ vs. $96 \pm 37 \mathrm{~ms})$, and a longer DSVRT 50 (25.0 $\pm 11.0 \%$ vs. $15.6 \pm 4.0 \%)$ (all $\mathrm{p}<0.05$ ). CMR diastolic parameters were independent predictors of TTE-based diastolic dysfunction after adjusting for left ventricular hypertrophy, hypertension, and coronary artery disease. Good correlations were observed between CMR PSRE $/ P S R_{A}$ and early-to-late diastolic annular velocity ratios ( $\left.e^{\prime} / a^{\prime}\right)$ measured by TDI ( $r=0.756$ to $\left.0.828, p<0.001\right)$.

Conclusions: 3D MA sweep volumes generated by semi-automated AVJ tracking in routinely acquired CMR images yielded diastolic parameters that were effective in identifying patients with diastolic dysfunction when correlated with TTE-based variables.

Keywords: Diastolic function, Mitral annulus, Cardiovascular magnetic resonance, Echocardiography, Feature-tracking

\footnotetext{
* Correspondence: Leon.Axel@nyumc.org

'Department of Radiology, Bernard and Irene Schwartz Center for Biomedical

Imaging, New York University School of Medicine, 660 First Avenue, Room 411, New York, NY 10016, USA

2Department of Medicine, Leon H. Charney Division of Cardiology, New York University School of Medicine, New York, NY, USA
} 


\section{Background}

Left ventricular (LV) diastolic dysfunction refers to mechanical abnormalities that impede effective volume filling during diastole. This is the main mechanism of heart failure with preserved ejection fraction (HFPEF), which accounts for up to $50 \%$ of overall cases of heart failure [1]. Despite the absence of systolic dysfunction, patients with HFPEF still experience high rates of morbidity and mortality [2]. Factors associated with diastolic dysfunction include hypertrophic, infiltrative, and dilated cardiomyopathies, advanced age, coronary artery disease (CAD), and systemic hypertension (HTN) [3]. Moreover, diastolic dysfunction often precedes systolic dysfunction, and is a sensitive indicator of early coronary and hypertensive heart disease [4]. As a result, the characterization of diastolic function, even in asymptomatic subjects, may have important implications for prognosis and treatment strategies.

Transthoracic echocardiography (TTE) is currently the standard method used in the noninvasive evaluation of diastolic function. Commonly, pulsed-wave Doppler of transmitral flow (TMF) is used, with pulmonary venous flow (PVF) and tissue Doppler imaging (TDI), to assess myocardial relaxation and filling pressures. However, TTE measurements have limitations, due to variability in sampling locations and ultrasound beam alignment [5]. In particular, TDI-derived velocities are position dependent, and may not accurately reflect global diastolic function in the presence of regional dysfunction [6]. One study demonstrated that the average of four annular site measurements is more accurate than a single site measurement [7]. While $\mathrm{x}$-ray computed tomography has also been introduced as a potential way to assess diastolic function [8], this is still investigational.

Cardiovascular magnetic resonance (CMR) has emerged as a promising alternative modality for quantifying diastolic dysfunction [9]. CMR's good spatial resolution, field of view, and range of tissue contrasts allow correlation of cardiac function with morphologic and tissue characteristics. Measurements analogous to TTE measurements, such as TMF, PVF, and myocardial tissue velocities, can be performed with velocity phase-contrast CMR, which has demonstrated excellent agreement with Doppler $[10,11]$. Similar agreement with TTE has also been reported for CMR derived LV volume-time curves [12,13]. In addition, myocardial tagging [14] methods have had promising results. However, despite recent advances, CMR-based methods for diastology remain in their infancy. The existing methods require prolonged imaging acquisition and post-processing times, preventing routine use of CMR in assessing diastolic function [15].

Mitral annular (MA) dynamics has been previously studied with various methods [16-18]; however, the relationship between CMR MA motion and diastolic function remains largely unexplored. In a prior study, a 2D manual tracking technique in assessing CMR MA motion had been reported [19]. In this paper, we aimed to expand on this prior technique and identify patients with diastolic dysfunction by using 3D MA sweep volumes calculated from routinely acquired long-axis cine-CMR images, comparing the results to TTE.

\section{Methods}

\section{Patient population and study design}

The research protocol was approved by the local Institutional Review Board. Study subjects were identified from retrospectively reviewed clinical data of all consecutive patients who underwent both clinically indicated TTE and CMR at New York University Langone Medical Center between June 2011 and December 2013. Inclusion criteria were: (1) cine-CMR performed within 2 months of TTE,

(2) TTE included assessment of diastolic function, and

(3) normal LV systolic function on both CMR and TTE (EF $>50 \%)$. Patients were excluded for: (1) incomplete CMR images for MA reconstruction, (2) poor CMR image quality due to arrhythmias (atrial fibrillation, ventricular ectopy), or (3) sinus tachycardia that rendered interpretation of the cardiac phases difficult [9]. Clinical data at the time of imaging were collected, including symptoms, CAD history, presence of structural heart disease on CMR, and cardiovascular risk factors such as HTN, hyperlipidemia, diabetes, and tobacco use. A positive history of CAD was defined as: reports of myocardial infarction, evidence of disease on diagnostic tests, or previous revascularization.

Study subjects were divided into normal diastolic function and diastolic dysfunction groups, using TDI velocities as reference. According to echocardiography guidelines [20,21], diastolic dysfunction was defined as septal e' MA peak velocity $<8 \mathrm{~cm} / \mathrm{s}$, lateral e' MA peak velocity $<10 \mathrm{~cm} / \mathrm{s}$, or maximal LA volume index $\geq 34 \mathrm{~mL} / \mathrm{m}^{2}$. Abnormal LA dilatation was defined as LA volume index $\geq 29 \mathrm{~mL} / \mathrm{m}^{2}[22]$.

\section{Imaging protocol \\ Echocardiography}

TTE was performed using conventional equipment: Philips iE33 (Phillips Medical Systems, MA, USA), General Electric Vivid 7 (General Electric Medical Systems, WI, USA), or Siemens SC2000 (Siemens Medical Solutions, CA, USA). Standard apical and parasternal views were obtained. Function and dimensional measurements were performed according to accepted guidelines [22]. TMF velocities were recorded with pulsed-wave Doppler sampling at the mitral valve leaflet tips in the apical 4chamber view. TDI was performed with pulsed-wave Doppler sampling at the MA junctions of the septal and lateral walls in the apical 4-chamber view. 


\section{CMR}

Conventional cine-CMR (2D steady state free precession pulse sequence) was performed using 1.5 or $3 \mathrm{~T}$ MRI systems (Avanto, Tim Trio, Siemens Medical Solutions, Erlangen, Germany) with a 6-element phased array anterior receiver coil, as part of routine clinical protocol. Cine images were acquired in multiplanar short-and long-axis views with retrospective electrocardiographic gating. Cine short-axis views were used for routine evaluation of global cardiac function. Typical imaging parameters were as follows: $\mathrm{TR} / \mathrm{TE}=2.4 / 1.4 \mathrm{~ms}$, temporal resolution $=37-63 \mathrm{~ms}$, in-plane spatial resolution $=1.4 \mathrm{~mm} \times 1.4 \mathrm{~mm}-1.8 \mathrm{~mm} \times$ $1.8 \mathrm{~mm}$, flip angle $=51^{\circ}$, slice thickness $=6 \mathrm{~mm}$, receiver bandwidth $=930 \mathrm{~Hz} /$ pixel, and 25 reconstructed phases per cardiac cycle.

\section{CMR image analysis}

\section{AVJ tracking and interpolated $3 D$ reconstruction of mitral annulus}

The atrioventricular junction (AVJ), the septal and lateral junction between the left atrium and ventricle, was tracked in two-, three-, and four-chamber longaxis CMR views over the cardiac cycle [19]. This was performed semi-automatically in lab-written MATLAB (MathWorks Inc., MA, USA) using normalized crosscorrelation (NCC), a well-known feature-tracking algorithm $[23,24]$ that has been used in MA location tracking in CMR [18]. Briefly, the user initially selects an AVJ point in the image of cardiac phase 1 (Figure 1A). This point becomes the center of a square template (red square) that undergoes NCC with a larger ROI (yellow square) in the image of phase 2 (Figure 1B), producing a correlation coefficient map (empirically, the use of a 20-pixel square in the initial phase and a 40-pixel square in the subsequent phase produced good tracking results that were not sensitive to the precise number of pixels used in this study).

In this calculated correlation map, the location of the maximum-intensity pixel indicates the best corresponding location of the initial template feature in the new ROI, which is thus taken as the AVJ location in phase 2 (Figure 1C). This new point becomes the center of a new template that then undergoes similar calculation of the NCC with the corresponding ROI in the next phase; the process is similarly automatically repeated for the subsequent phases, in order to track the AVJ locations throughout the cardiac cycle. The software also allows for optional interactive user correction of the AVJ location, should there be tracking errors (e.g., due to blurring artifacts). After user correction for a specific phase, if needed, automated AVJ tracking with the NCC method is then performed again for the subsequent phases in the image series, using the corrected location as the starting point.

For each cardiac phase, two AVJ locations were tracked in each of the two-, three-, and four-chamber long-axis CMR views (Figure 2A), creating six independent spatial $3 \mathrm{D}$ coordinates within the AVJ that were tracked over the cardiac cycle. Note that $2 \mathrm{D}$ image coordinates were transformed into the corresponding 3D space coordinates, using spatial information about the image acquisition locations from DICOM headers. A 3D spline curve was then used to interpolate these 6 distinct 3D spatial coordinates sampled within the MA at each cardiac cycle phase, in order to create a reconstruction of the MA in $3 \mathrm{D}$ space (Figure 2B), using solid-modeling software Rhinoceros

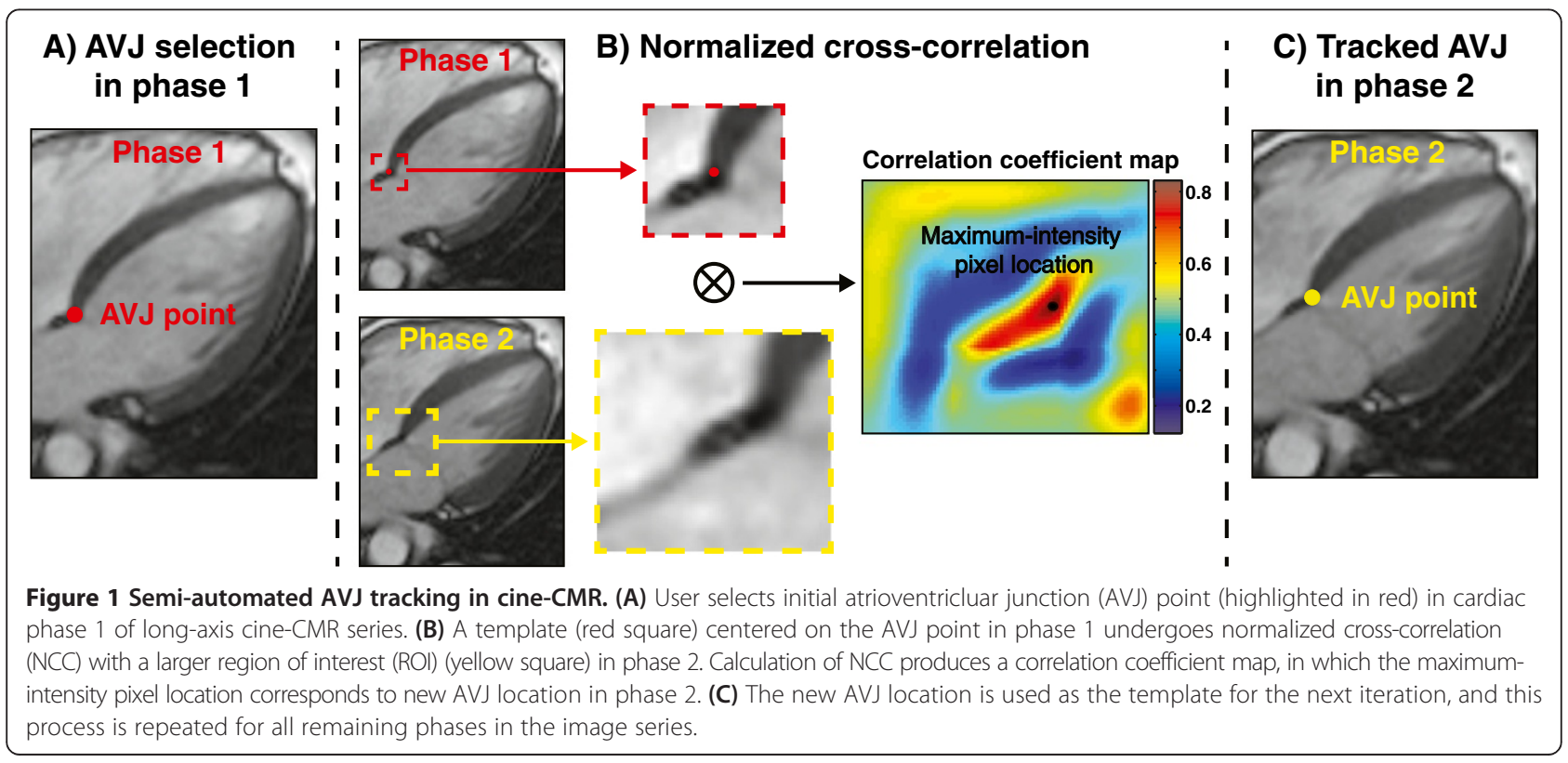




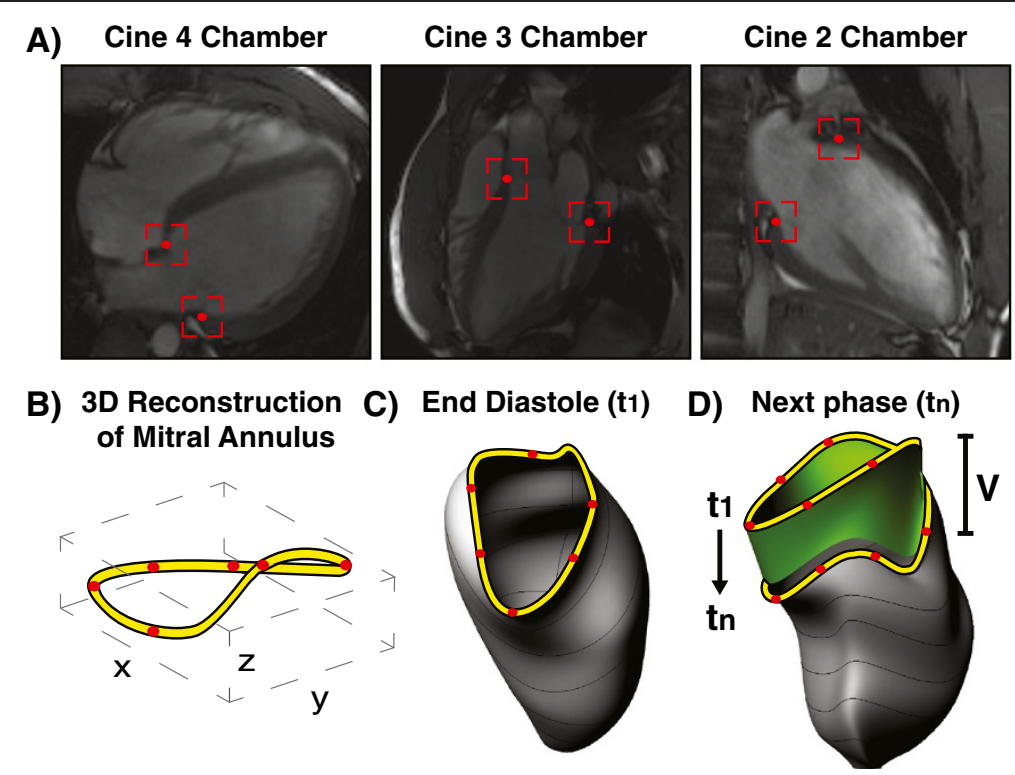

Figure 2 Interpolated 3D reconstruction of mitral annulus. (A) AVJ points were tracked in two-, three-, and four-chamber cine-CMR views to create six distinct spatial points (highlighted in red) sampled in the mitral annulus (MA) per cardiac phase. (B) 3D spline interpolation was applied to the 3D space locations of these points to create a 3D reconstruction of the mitral annulus (MA). (C, D) A 3D MA sweep volume (V, highlighted in green) was generated for each cardiac phase relative to the MA area at $t_{1}$ (end diastole), by summing incremental volumes calculated from the MA area at that phase and the distance the MA traversed from the previous phase.

(McNeel, WA, USA). This is analogous to methods that have been used to reconstruct the $3 \mathrm{D}$ shape of the MA from ultrasound images [25]. 3D spline curves have been similarly used to reconstruct the $3 \mathrm{D}$ MA structure in prior CMR studies [18,26]. A 3D MA incremental sweep volume $\left(V_{n}\right)$ was then generated for each cardiac phase $t_{n}$, using the MA areas at $t_{n-1}$ and $t_{n}$, and the $3 D$ distance (positive or negative) through which the MA traversed (Figure 2C, D); the net sweep volume at a given cardiac phase was derived from the sum of the incremental volumes starting from end-diastole.

\section{Diastolic function}

The resultant MA sweep volume curve was manually divided into its corresponding cardiac cycle intervals according to transitions in its slope: systole, early diastole (ED), mid-diastole (MD), and atrial systole (AS) (Figure 3). In addition, its first derivative was calculated to characterize the MA sweep volume rate in each interval. For comparisons between different heart sizes, which may confound the absolute amounts of myocardial relaxation, the sweep volumes (and rates) were normalized to the corresponding end systolic sweep volume (ESSV). To quantify measures related to LV myocardial relaxation, the following CMR diastolic parameters were derived from the curves: (1) peak sweep rates (PSR) in ED and AS; (2) average sweep rates in ED, MD, AS; (3) percentage sweep volume recovery in ED and AS, defined by the proportion of ESSV that the MA had recovered during the given interval; and (4) the ratio of peak sweep rate in ED to peak sweep rate in $\mathrm{AS}\left(\mathrm{PSR}_{\mathrm{E}} / \mathrm{PSR}_{\mathrm{A}}\right)$. Note that $\mathrm{PSR}_{\mathrm{E}} / \mathrm{PSR}_{\mathrm{A}}$ is analogous to the $\mathrm{e}^{\prime} / \mathrm{a}^{\prime}$ ratio used in TDI.

Three time-interval parameters were selected to characterize diastolic function from the sweep volumes: (1) acceleration time $\left(\mathrm{AT}_{\mathrm{SV}}\right)$, measured from $\mathrm{ED}$ onset to the time of $\mathrm{PSR}_{\mathrm{E}}$; $(2)$ deceleration time $\left(\mathrm{DT}_{\mathrm{SV}}\right)$, measured by linear extrapolation of $\mathrm{PSR}_{\mathrm{E}}$ to baseline; and (3) $50 \%$ diastolic sweep volume recovery (DSVRT $\mathrm{D}_{50}$ ), defined as the time required in diastole for the MA to recover $50 \%$ of its end systolic sweep volume (adjusted for RR interval); this index had been introduced in a prior study to describe CMR LV volume filling [12].

\section{Statistical analysis}

All statistical analyses were performed using SPSS version 20.0 (SPSS Inc., Chicago, Illinois). Continuous variables were presented as mean $\pm \mathrm{SD}$, whereas categorical data were presented as frequencies with percentages. Differences between groups were evaluated using either the Student t-test for normally distributed data, or the Mann-Whitney U-test for asymmetrically distributed data; Shapiro-Wilk test was used to assess normality of distributions. Fisher's exact test was used to compare differences in categorical data. In addition, the association between CMR- and TTE-based parameters was assessed using the Spearman's rank correlation test. For classification performance, receiver operating characteristic (ROC) analyses were used to evaluate the ability of CMR parameters 
A)

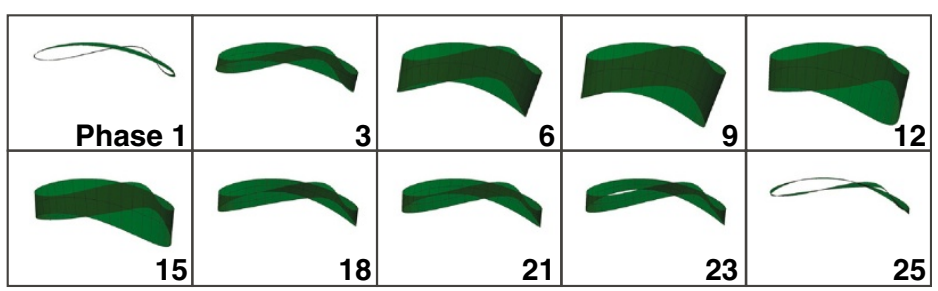

B)

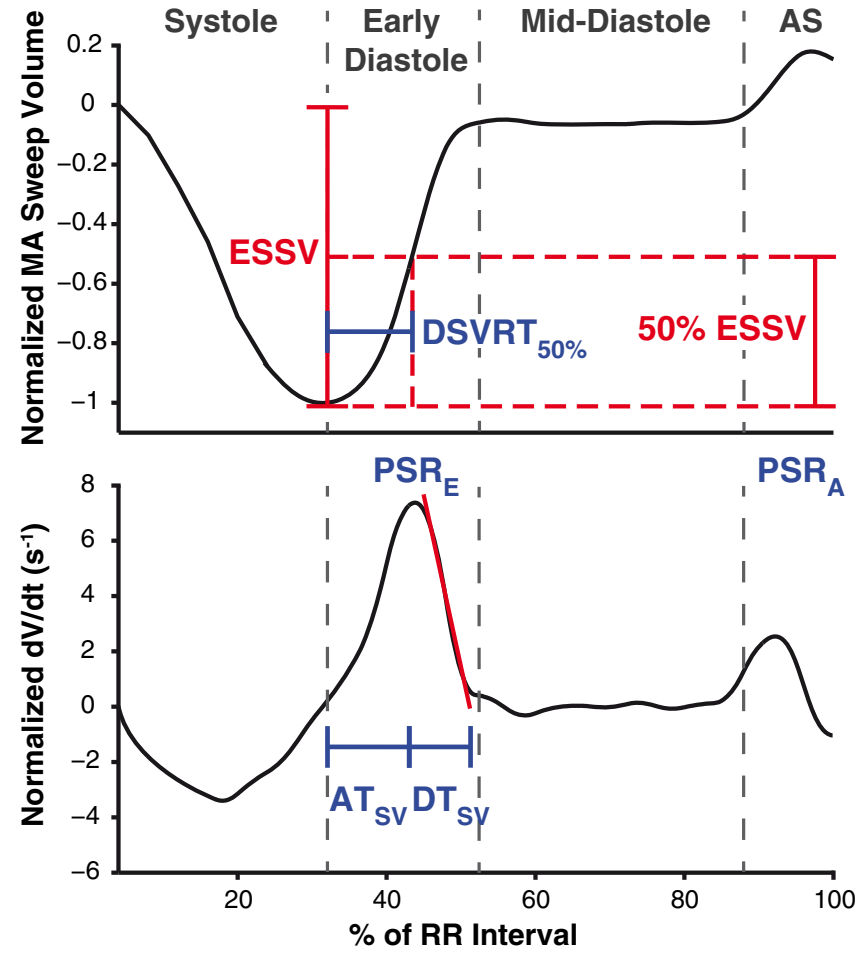

Figure 3 Mitral annulus sweep volume curve and associated diastolic parameters. (A) Representative 3D models of MA sweep volumes at different phases of the cardiac cycle. (B) Representative MA sweep volume (upper) and sweep rate (lower) profiles from a subject with normal diastolic function. Both curves were normalized to end-systolic sweep volume (ESSV). Cardiac intervals were identified based on the transitions in slope of the sweep volume curve, distinguishing systole, early diastole, mid-diastole, and atrial systole (AS). 50\% diastolic sweep volume recovery time (DSVRT ${ }_{50}$ ) was measured as the time required in diastole for the MA to recover 50\% of ESSV, and was adjusted for RR interval. Normalized peak sweep rates in early diastole $\left(P S R_{E}\right)$ and atrial systole $\left(P_{S} R_{A}\right)$ are shown in the sweep rate curve. Sweep volume acceleration time $\left(A_{S V}\right)$ was measured from onset of early diastole to the time of PSR $R_{E}$ and sweep volume deceleration time $\left(D T_{S V}\right)$ was measured by linear extrapolation of PSR to baseline.

to identify patients with TTE-based diastolic dysfunction. Optimal cutoff values for these parameters were obtained, along with their associated sensitivity and specificity. Multivariate logistic regression was used to determine the predictive ability of CMR parameters with respect to TTE-based outcomes, after controlling for age, HTN, left ventricular hypertrophy (LVH), and CAD status. A two-tailed value of $\mathrm{p}<0.05$ was considered statistically significant.

Intraobserver and interobserver variability were evaluated for 10 randomly selected subjects using intraclass correlation coefficients (ICC), Bland-Altman analyses, and Pearson's $r$ correlation coefficients. For interobserver variability assessment, analyses were performed by two independent CMR readers who were blinded to TTE and each other's results. For intraobserver variability assessment, analyses were performed by one user who re-analyzed the same 10 subjects after a 3 week interval.

\section{Results}

\section{Population characteristics}

A total of 124 consecutive subjects met the selection criteria and were included in the study. The time difference between the dates of CMR and TTE was $16 \pm$ 16 days. Based on TTE findings, subjects were classified as either having normal diastolic function $(n=62)$ or diastolic dysfunction $(n=62)$.

Table 1 details the baseline characteristics of the study population. Compared to the normal diastolic function group, those with TTE-based diastolic dysfunction were 
Table 1 Population characteristics

\begin{tabular}{|c|c|c|c|c|}
\hline & Overall & Normal diastolic function $\neq(n=62)$ & Diastolic dysfunction $\neq(n=62)$ & $\mathbf{P}$ \\
\hline \multicolumn{5}{|l|}{ Clinical } \\
\hline Age, years & $51 \pm 17$ & $40 \pm 14$ & $61 \pm 14$ & $<0.001^{*}$ \\
\hline Male, n (\%) & 76 & $31(49 \%)$ & $45(65 \%)$ & $0.046^{*}$ \\
\hline Heart Rate, bpm & $71 \pm 12$ & $72 \pm 12$ & $71 \pm 12$ & 0.628 \\
\hline \multicolumn{5}{|l|}{ Cardiovascular risk factors } \\
\hline Hypertension, n (\%) & 54 & $16(26 \%)$ & $38(61 \%)$ & $<0.001^{*}$ \\
\hline Hyperlipidemia, n (\%) & 52 & $17(27 \%)$ & $35(56 \%)$ & $0.002^{*}$ \\
\hline Diabetes mellitus, n (\%) & 9 & $3(5 \%)$ & $6(10 \%)$ & 0.491 \\
\hline Tobacco use, n (\%) & 45 & 19 (31\%) & $26(42 \%)$ & 0.262 \\
\hline Coronary artery disease, n (\%) & 29 & $7(11 \%)$ & $22(35 \%)$ & $0.003^{*}$ \\
\hline \multicolumn{5}{|l|}{ Cardiovascular symptoms } \\
\hline Angina, n (\%) & 32 & $18(29 \%)$ & $14(23 \%)$ & 0.539 \\
\hline Dyspnea, n (\%) & 28 & $9(15 \%)$ & 19 (31\%) & $0.052 \dagger$ \\
\hline Palpitation/Arrhythmia, n (\%) & 29 & $15(24 \%)$ & $14(23 \%)$ & 1.000 \\
\hline Syncope/Pre-syncope, n (\%) & 20 & $10(16 \%)$ & $10(16 \%)$ & 1.000 \\
\hline Asymptomatic, n (\%) & 35 & $20(32 \%)$ & $15(24 \%)$ & 0.425 \\
\hline \multicolumn{5}{|l|}{ Structural heart disease on CMR } \\
\hline Normal, n (\%) & 57 & $42(68 \%)$ & $15(24 \%)$ & $<0.001^{*}$ \\
\hline Ischemic heart disease, n (\%) & 10 & $3(5 \%)$ & $7(11 \%)$ & 0.323 \\
\hline Congenital heart disease, n (\%) & 4 & $3(5 \%)$ & $1(2 \%)$ & 0.619 \\
\hline Infiltrative disease, n (\%) & 5 & $1(2 \%)$ & $4(6 \%)$ & 0.365 \\
\hline LV hypertrophy, n (\%) & 30 & $6(10 \%)$ & $24(39 \%)$ & $<0.001^{*}$ \\
\hline Dilated cardiomyopathy, n (\%) & 2 & $0(0 \%)$ & $2(3 \%)$ & 0.496 \\
\hline Inflammatory cardiomyopathy, n (\%) & 9 & $5(8 \%)$ & $4(6 \%)$ & 1.000 \\
\hline \multicolumn{5}{|l|}{ Function measures by TTE } \\
\hline Interventricular septal wall thickness, mm & $1.11 \pm 0.39$ & $0.96 \pm 0.32$ & $1.25 \pm 0.41$ & $<0.001^{*}$ \\
\hline Inferolateral wall thickness, mm & $0.98 \pm 0.18$ & $0.9 \pm 0.15$ & $1.06 \pm 0.17$ & $<0.001^{*}$ \\
\hline LV end-diastolic diameter, mm & $4.52 \pm 0.6$ & $4.69 \pm 0.54$ & $4.35 \pm 0.61$ & $0.001 *$ \\
\hline LV end-systolic diameter, mm & $2.86 \pm 0.52$ & $2.94 \pm 0.49$ & $2.78 \pm 0.55$ & 0.102 \\
\hline LV mass index, $\mathrm{g} / \mathrm{m}^{2}$ & $87 \pm 27$ & $80 \pm 21$ & $95 \pm 31$ & $0.010^{*}$ \\
\hline LV ejection fraction,\% & $64 \pm 6$ & $62 \pm 6$ & $65 \pm 7$ & $0.059+$ \\
\hline $\mathrm{LA}$ volume index, $\mathrm{ml} / \mathrm{m}^{2}$ & $25 \pm 9$ & $22 \pm 7$ & $27 \pm 10$ & $0.001 *$ \\
\hline LA dilatation, n (\%) & 30 & $6(10 \%)$ & $24(39 \%)$ & $<0.001^{*}$ \\
\hline \multicolumn{5}{|l|}{ Function measures by CMR } \\
\hline LV end-diastolic volume, mL & $152 \pm 39$ & $157 \pm 40$ & $147 \pm 39$ & 0.211 \\
\hline LV end-systolic volume, $\mathrm{mL}$ & $59 \pm 20$ & $62 \pm 19$ & $57 \pm 20$ & 0.234 \\
\hline Stroke volume, mL & $93 \pm 24$ & $95 \pm 24$ & $90 \pm 24$ & 0.242 \\
\hline Cardiac output, L/min & $7.4 \pm 8.7$ & $8.5 \pm 12$ & $6.2 \pm 2$ & 0.241 \\
\hline Cardiac index, $\mathrm{L} / \mathrm{min} / \mathrm{m}^{2}$ & $3.3 \pm 0.9$ & $3.4 \pm 0.9$ & $3.2 \pm 0.9$ & 0.101 \\
\hline LV ejection fraction,\% & $61 \pm 6$ & $61 \pm 5$ & $62 \pm 7$ & 0.720 \\
\hline
\end{tabular}

Data expressed as mean \pm SD or frequencies (percentages). $L V=$ Left ventricular; $L A=$ Left atrial. ${ }^{*} \mathrm{P}<0.05,+\mathrm{P}<0.10$, $\neq$ Patient classification based on echocardiography reference. 
older in age $(61 \pm 14$ vs. $40 \pm 14$ years, $\mathrm{p}<0.001)$ and were more likely to be male $(p=0.046)$. Clinically, the diastolic dysfunction group had increased frequencies of HTN (61\% vs. $26 \%, \mathrm{p}<0.001$ ), hyperlipidemia (56\% vs. $27 \%, \mathrm{p}=0.002)$, and $\mathrm{CAD}(35 \%$ vs. $11 \%, \mathrm{p}=0.003)$. In terms of symptoms, patients with diastolic dysfunction were more likely to complain of dyspnea ( $31 \%$ vs. $15 \%$, $\mathrm{p}=0.052$ ). A higher proportion of subjects in the diastolic dysfunction group, compared to those in the normal diastolic function group, had CMR evidence of structural heart disease (76\% vs. 32\%, p < 0.001); in particular, the prevalence of LVH was significantly higher in the diastolic dysfunction group ( $39 \%$ vs. $10 \%, \mathrm{p}<0.001)$. These clinical findings were consistent with prior reports on the prevalence of diastolic dysfunction [27]. Morphologically, TTE-derived LV mass index and LA volume index were both significantly higher in the diastolic dysfunction group.

\section{AVJ tracking and MA reconstruction}

Our CMR method successfully generated MA sweep volume curves for all subjects in the study. The approximate processing time for each case was less than 3 minutes. The processing included the initial user delineation of AVJ points, review and possible correction of semi-automated tracking results, MA reconstruction in $3 \mathrm{D}$, and the identification of cardiac intervals within the sweep volume curve. Manual corrections of AVJ locations were primarily necessary in images with blurring artifacts affecting the regions of interest. Approximately $30 \%$ of cine image series required at least one manual correction. Cases that did not require manual correction were analyzed in under 1 minute.

Figure 4A illustrates representative patients, comparing the normalized MA sweep volume curves and their derivatives between subjects with normal diastolic function and diastolic dysfunction. These curves show that the MA of a diastolic dysfunction subject requires more time to recover from its end systolic volume state, as quantified by the parameter DSVRT ${ }_{50}$. Furthermore, the derivative curve provides a visual representation of the normalized MA sweep rate at each cardiac interval. In normal diastolic function, there was typically a higher MA sweep rate during early diastole that was accompanied by a relatively lower sweep rate during atrial systole; this was reversed in diastolic dysfunction. In these cases, peak MA sweep rates $\left(\mathrm{PSR}_{\mathrm{E}}\right.$ and $\mathrm{PSR}_{\mathrm{A}}$ ) on CMR were in qualitative relative agreement with the echocardiographically-derived early $\left(e^{\prime}\right)$ and late $\left(a^{\prime}\right)$ TDI annular velocity profiles (Figure 4B). In addition, the utility of $\mathrm{DT}_{\mathrm{SV}}$ was demonstrated in the sweep volume derivative curve, where the diastolic dysfunction subjects showed a relative flattening of the deceleration component in early diastole.

\section{LV diastolic function}

Table 2 describes the CMR and TTE diastolic parameters for the two groups. Compared to patients with TTE-based normal diastolic function, those with TTE-based diastolic dysfunction had slower normalized peak and average MA sweep rates in early diastole $\left(5.3 \pm 1.4 \mathrm{~s}^{-1}\right.$ vs. $7.7 \pm 1.7 \mathrm{~s}^{-1}$ and $2.5 \pm 0.6 \mathrm{~s}^{-1}$ vs. $4.2 \pm 1.0 \mathrm{~s}^{-1}$, respectively, both $\mathrm{p}<0.001)$. The diastolic dysfunction group also had a lower $\mathrm{PSR}_{\mathrm{E}} / \mathrm{PSR}_{\mathrm{A}}$ ratio $(0.9 \pm 0.4$ vs. $1.8 \pm 0.7, \mathrm{p}<0.001)$, due to their relatively higher peak sweep rate in atrial systole. During early diastole, the MA of hearts with diastolic dysfunction recovered a smaller proportion of their end systolic sweep volume $(54 \pm 12 \%$ vs. $69 \pm 10 \%, \mathrm{p}<0.001)$, and their MA sweep volume constituted a smaller percentage of their stroke volume $(7.6 \pm 2.2 \%$ vs. $11.2 \pm 2.9 \%$, $\mathrm{p}<0.001)$. Diastolic dysfunction was also differentiated by its longer $\mathrm{DT}_{\mathrm{SV}}(144 \pm 55 \mathrm{~ms}$ vs. $96 \pm 37 \mathrm{~ms}, \mathrm{p}<0.001)$ and longer DSVRT ${ }_{50}(25 \pm 11 \%$ vs. $16 \pm 4.0 \%, \mathrm{p}<0.001)$.

Figure 5 illustrates the correlations of MA velocities between CMR and TDI measured at different locations. Although there was a strong positive correlation between CMR PSR $/ \mathrm{PSR}_{\mathrm{A}}$ and echo septal e' $/ \mathrm{a}^{\prime} \quad(\mathrm{r}=0.756$, $\mathrm{p}<0.001)$, the correlation between $\mathrm{PSR}_{\mathrm{E}} / \mathrm{PSR}_{\mathrm{A}}$ and lateral $\mathrm{e}^{\prime} / \mathrm{a}^{\prime}$ was even stronger $(\mathrm{r}=0.803, \mathrm{p}<0.001)$. The strongest correlation, however, was seen between $\mathrm{PSR}_{\mathrm{E}} /$ $\mathrm{PSR}_{\mathrm{A}}$ and the average $\mathrm{e}^{\prime} / \mathrm{a}^{\prime}$ of septal and lateral positions $(\mathrm{r}=0.828, \mathrm{p}<0.001)$.

\section{Classification performance of CMR parameters}

Table 3 summarizes the ROC analysis of CMR diastolic parameters in the identification of TTE-based diastolic dysfunction. Based on areas under the curve (AUC), $\mathrm{PSR}_{\mathrm{E}}$ and $\mathrm{PSR}_{\mathrm{E}} / \mathrm{PSR}_{\mathrm{A}}$ ratio demonstrated excellent classification power (both $\mathrm{AUC}=0.88)$, as did $\mathrm{DT}_{\mathrm{SV}}(\mathrm{AUC}=0.83$ ) and $\operatorname{DSVRT}_{50}(\mathrm{AUC}=0.79)$. The optimal cutoff value for $\mathrm{PSR}_{\mathrm{E}}$ was $6.7 \mathrm{~s}^{-1}$, with a sensitivity of $89 \%$ and specificity of $74 \%$; the optimal cutoff for $\mathrm{PSR}_{\mathrm{E}} / \mathrm{PSR}_{\mathrm{A}}$ was 1.17 , with a sensitivity of $84 \%$ and specificity of $81 \%$. In comparison, $\mathrm{DT}_{\mathrm{SV}}$ and $\mathrm{DSVRT}_{50}$ exhibited slightly lower accuracies, with optimal cutoffs at $101 \mathrm{~ms}$ and $19.45 \%$, respectively. A recovery threshold of $50 \%$ of end-systolic sweep volume was chosen for the parameter DSVRT $_{50}$ because this generated the best classification performance compared to other recovery thresholds (thresholds of $40 \%, 60 \%, 70 \%$, and $80 \%$ produced $A U C ' s$ ranging from 0.60 to 0.75 ).

\section{CMR prediction of diastolic dysfunction}

Multivariate logistic regression analysis demonstrated that $\mathrm{PSR}_{\mathrm{E}}, \mathrm{PSR}_{\mathrm{E}} / \mathrm{PSR}_{\mathrm{A}}$, and $\mathrm{DSVRT}_{50}$ were significant independent predictors of TTE-based diastolic dysfunction after controlling for age, HTN, LVH, and CAD status (Table 4). $\mathrm{DT}_{\mathrm{SV}}$ was near significant as an independent predictor with an odds ratio of 1.12 per 10 ms increment $(\mathrm{p}=0.077)$. In addition, $\mathrm{PSR}_{\mathrm{E}} / \mathrm{PSR}_{\mathrm{A}}$ had an odds ratio of 
A) CMR
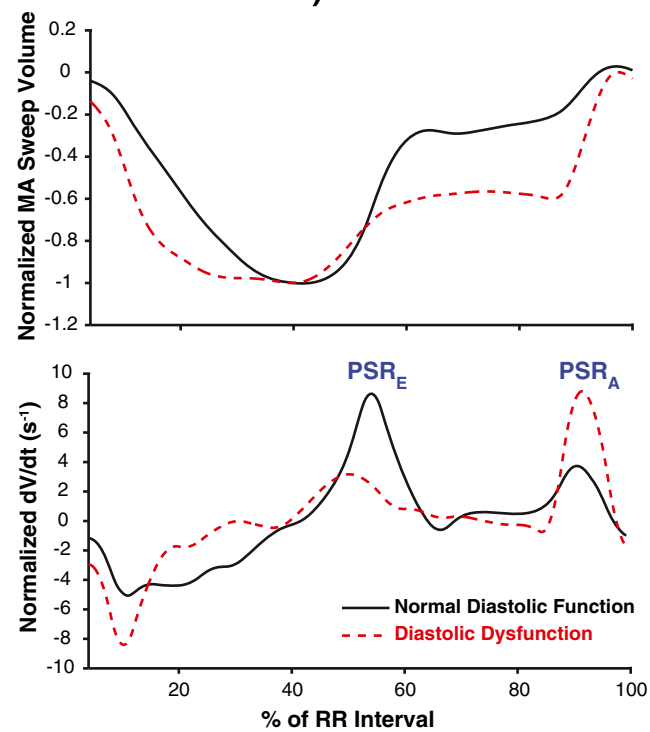

B) Tissue Doppler Imaging

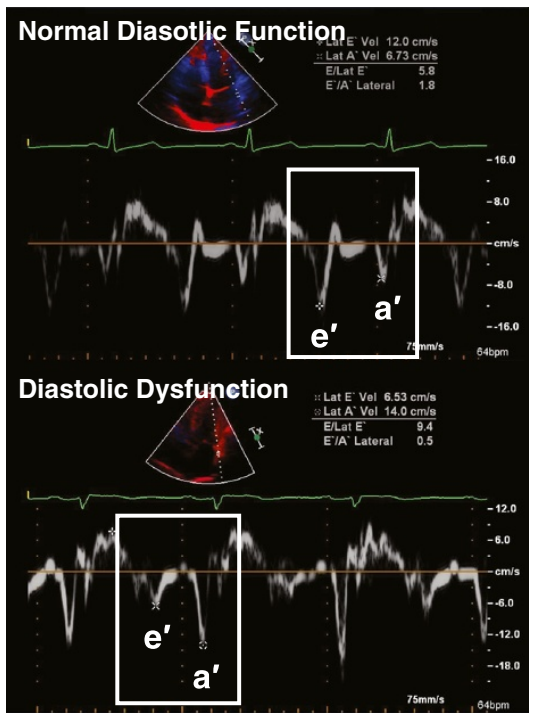

Figure 4 Graphical comparisons between normal diastolic function and diastolic dysfunction in diastole. (A) Representative MA sweep volume and sweep rate profiles of patients with TTE-based normal diastolic function (solid line) and diastolic dysfunction (dashed line) as generated by the proposed CMR method. Apparent differences in relative diastolic indices are seen in normalized peak sweep rates during early diastole (PSRE) and atrial systole $\left(\mathrm{PSR}_{\mathrm{A}}\right)$. (B) Corresponding tissue Doppler imaging, showing qualitatively similar relative lateral MA velocity profiles between the same two patients. Note that the patient with diastolic dysfunction had a decreased early diastolic annular velocity ( $\left.e^{\prime}\right)$ but an increased atrial systole velocity ( $a^{\prime}$ ), resulting in e'/a' reversal that is typical of diastolic dysfunction.

0.86 per 0.1 increment $(\mathrm{p}=0.016)$, whereas $\mathrm{PSR}_{\mathrm{E}}$ and DSVRT $_{50}$ had odds ratios of $0.54(\mathrm{p}=0.007)$ and 1.14 ( $\mathrm{p}=0.013)$, respectively.

CMR diastolic parameters were used as covariates to construct predictive models with logistic regression. A model including both $\mathrm{PSR}_{\mathrm{E}}$ and $\mathrm{PSR}_{\mathrm{E}} / \mathrm{PSR}_{\mathrm{A}}$ showed an improved AUC of 0.895 in predicting TTE-based diastolic dysfunction. When adding in $\mathrm{DT}_{\mathrm{SV}}$ and $\mathrm{DSVRT}_{50}$, the AUC improved slightly to 0.896 . The latter model with all four parameters was used to demonstrate the prevalence of CMR-based diastolic dysfunction in a number of important clinical conditions in our sample population (Figure 6). As shown, the prevalence of CMR-based diastolic dysfunction was almost twice as great in patients with LVH compared to patients without LVH $(\mathrm{p}=0.002)$. Similar results were observed in patients with HTN and CAD. In addition, the same predictive model showed that LA dilatation was seen in 24 of 65 subjects (37\%) with CMR-based diastolic dysfunction and 6 of 59 (10\%) subjects with CMR-based normal diastolic function $(\mathrm{p}<0.001)$. Based on ROC analyses, LA volume index demonstrated only moderate classification powers in predicting both CMR-based and TTE-based diastolic dysfunction ( $\mathrm{AUC}=0.64, \mathrm{AUC}=0.67$, respectively).

\section{Reproducibility}

Table 5 shows the intraobserver and interoberserver variabilities using ICC, the Bland-Altman test, and Pearson's r correlation. CMR diastolic parameters demonstrated good consistency in terms of correlations (ICC ranged from 0.88 to 0.97 ) with minimal degree of biases for both intraobserver and interobserver measurements.

\section{Discussion}

This study demonstrates that CMR diastolic parameters derived from 3D MA sweep volumes were reproducible and could accurately differentiate between patients with normal diastolic function and diastolic dysfunction, as established by TDI. CMR-based measurements of peak sweep rates were also strongly correlated with analogous TDI velocity indices. Diastolic assessments using CMR 3D MA sweep volumes were validated in a diverse population of 124 subjects with normal systolic function measures, which revealed an increased prevalence of CMR based diastolic dysfunction in patients with HTN, CAD, and LVH. This suggests that MA sweep volume may contribute to the evaluation of LV diastolic function, potentially providing additional prognostic information and guidance that could be useful in management before frank heart failure occurs [28].

Our method addresses several limitations encountered by current CMR methods of assessing diastolic function. Notably, routine use of phase-contrast imaging and tagging have been hindered by the need for additional imaging pulse-sequences and associated acquisition time. In addition, manual calculation of LV volumetric curves 
Table 2 Diastolic parameters

\begin{tabular}{|c|c|c|c|}
\hline & Normal diastolic function $\neq(n=62)$ & Diastolic dysfunction $\neq(n=62)$ & $\mathbf{P}$ \\
\hline \multicolumn{4}{|l|}{ CMR mitral annulus sweep volume } \\
\hline Normalized peak sweep rate, early diastole $\left(\mathrm{PSR}_{\mathrm{E}}\right), \mathrm{s}^{-1}$ & $7.72 \pm 1.7$ & $5.25 \pm 1.38$ & $<0.001^{*}$ \\
\hline Peak sweep rate, early diastole, $\mathrm{cm}^{3} / \mathrm{s}$ & $117 \pm 43$ & $65 \pm 28$ & $<0.001^{*}$ \\
\hline Normalized peak sweep rate, atrial systole $\left(\mathrm{PSR}_{\mathrm{A}}\right), \mathrm{s}^{-1}$ & $4.67 \pm 1.38$ & $6.56 \pm 1.99$ & $<0.001^{*}$ \\
\hline Peak sweep rate, atrial systole, $\mathrm{cm}^{3} / \mathrm{s}$ & $70 \pm 24$ & $80 \pm 29$ & $0.031^{*}$ \\
\hline $\mathrm{PSR}_{\mathrm{E}} / \mathrm{PSR}_{\mathrm{A}}$ ratio & $1.82 \pm 0.69$ & $0.9 \pm 0.44$ & $<0.001^{*}$ \\
\hline Percentage sweep volume recovery, early diastole & $69.09 \pm 10.02$ & $54.29 \pm 12.37$ & $<0.001^{*}$ \\
\hline Absolute sweep volume, early diastole, $\mathrm{cm}^{3}$ & $10.64 \pm 4.1$ & $6.83 \pm 2.66$ & $<0.001^{*}$ \\
\hline Absolute sweep volume/stroke volume, early diastole, \% & $11.17 \pm 2.89$ & $7.6 \pm 2.21$ & $<0.001^{*}$ \\
\hline Percentage sweep volume recovery, atrial systole & $33.78 \pm 9.92$ & $49.1 \pm 13.76$ & $<0.001^{*}$ \\
\hline Absolute sweep volume, atrial systole, $\mathrm{cm}^{3}$ & $5.03 \pm 1.72$ & $6.01 \pm 2.35$ & $0.014^{*}$ \\
\hline Absolute sweep volume/stroke volume, atrial systole, \% & $5.41 \pm 1.78$ & $6.81 \pm 2.24$ & $0.014^{*}$ \\
\hline Normalized average sweep rate, early diastole, $\mathrm{s}^{-1}$ & $4.2 \pm 1.02$ & $2.53 \pm 0.63$ & $<0.001^{*}$ \\
\hline Normalized average sweep rate, mid-diastole, $\mathrm{s}^{-1}$ & $0.36 \pm 0.71$ & $0.87 \pm 1.34$ & $0.019 *$ \\
\hline Normalized average sweep rate, atrial systole, $\mathrm{s}^{-1}$ & $2.67 \pm 0.94$ & $3.88 \pm 1.26$ & $<0.001^{*}$ \\
\hline Sweep volume deceleration time (DT $\left.{ }_{\text {sv }}\right)$, ms & $96.25 \pm 36.56$ & $144.19 \pm 54.89$ & $<0.001^{*}$ \\
\hline Sweep volume acceleration time (ATsv), ms & $102.48 \pm 35.03$ & $114.2 \pm 41.09$ & $0.066+$ \\
\hline $50 \%$ Diastolic sweep volume recovery time (DSVRT $\left.{ }_{50}\right), \%$ & $15.58 \pm 4$ & $24.97 \pm 11.03$ & $<0.001^{*}$ \\
\hline \multicolumn{4}{|l|}{ Echocardiography } \\
\hline $\mathrm{E}, \mathrm{cm} / \mathrm{s}$ & $84.47 \pm 22.22$ & $75.81 \pm 25.08$ & $0.020^{*}$ \\
\hline $\mathrm{A}, \mathrm{cm} / \mathrm{s}$ & $59.45 \pm 17.75$ & $69.9 \pm 22.7$ & $0.005^{*}$ \\
\hline$E / A$ & $1.51 \pm 0.51$ & $1.22 \pm 0.67$ & $<0.001^{*}$ \\
\hline Deceleration time, ms & $219 \pm 48$ & $230 \pm 47$ & 0.200 \\
\hline$e^{\prime}$ septal, cm/s & $10.14 \pm 2.22$ & $6.05 \pm 1.4$ & $<0.001^{*}$ \\
\hline$a^{\prime}$ septal, cm/s & $8.25 \pm 1.89$ & $8.98 \pm 2.44$ & $0.064 \dagger$ \\
\hline e'/a' septal & $1.3 \pm 0.41$ & $0.75 \pm 0.34$ & $<0.001^{*}$ \\
\hline $\mathrm{e}^{\prime}$ lateral, cm/s & $14.59 \pm 3.95$ & $8.1 \pm 2.56$ & $<0.001^{*}$ \\
\hline$a^{\prime}$ lateral, cm/s & $9.51 \pm 2.95$ & $10.4 \pm 3.75$ & 0.141 \\
\hline$e^{\prime} / a^{\prime}$ lateral & $1.7 \pm 0.71$ & $0.89 \pm 0.44$ & $<0.001 *$ \\
\hline E/e' (average) & $7.1 \pm 2.02$ & $11.47 \pm 6.26$ & $<0.001^{*}$ \\
\hline
\end{tabular}

Data expressed as mean \pm SD.

Peak and average sweep rates were normalized to end-systolic sweep volume. Percentage sweep volume recovery measured the proportion of recovery in reference to end-systolic sweep volume. $50 \%$ diastolic sweep volume recovery time was normalized to RR interval.

$\mathrm{E}=$ peak early mitral inflow velocity; $\mathrm{A}=$ peak late mitral inflow velocity; $\mathrm{e}^{\prime}=$ early diastolic mitral annular velocity; $\mathrm{a}^{\prime}=$ late diastolic mitral annular velocity; $\mathrm{MA}=$ mitral annulus.

${ }^{*} P<0.05,+P<0.10, \neq$ Patient classification based on echocardiography reference.

requires extensive post-processing effort and has associated subjectivity, while automatic measurements of these curves require proprietary and complex image-processing algorithms [13]. Our novel CMR method may provide a more practical option, since it assesses diastolic function by using conventional cine-CMR long-axis images that are already routinely acquired in clinical CMR examinations, without the need for additional imaging. This also enables users to analyze pre-existing cases.

To facilitate the measurement of MA sweep volumes, AVJ tracking was performed semi-automatically, using a simple NCC feature-tracking algorithm. NCC is an imageprocessing technique that has been used for many applications in motion tracking. NCC provides a means to assess the degree of similarity ("correlation") between two images, as a function of pixel position [23]. Assuming that the immediate surroundings of a given point in an initial image provide the features of a template, the algorithm "slides" and centers this template at each pixel within a neighborhood of this point in a subsequent image, and a correlation coefficient is calculated between the template and the subsequent image for each such pixel 

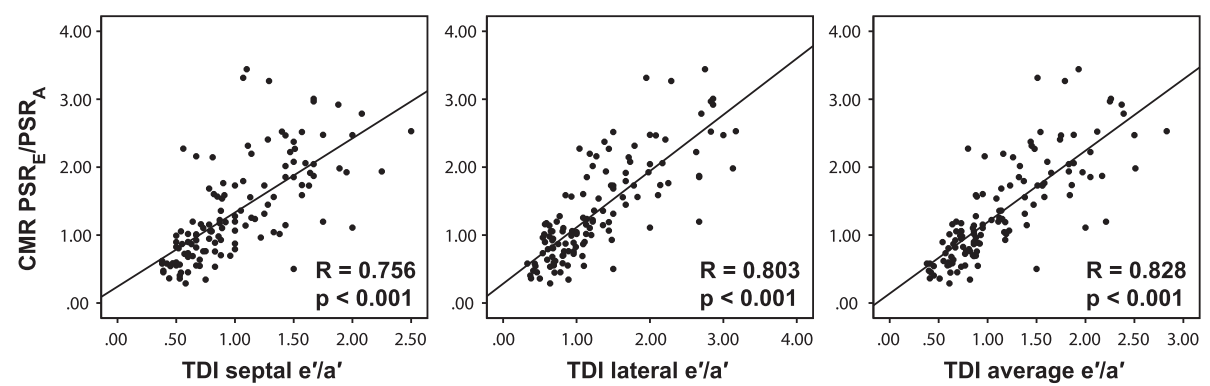

Figure 5 Correlation between CMR and TDI parameters. Spearman's rank test showed good positive correlations between CMR normalized peak volume sweep rates and TDI MA velocities in early-to-late diastolic ratios. CMR PSRE/PSR correlated better with TDI lateral e'/a' than it did with TDI septal $e^{\prime} / a^{\prime}$. However, $P S R_{E} / P S R_{A}$ correlated best with the average $e^{\prime} / a^{\prime}$ of lateral and septal locations.

position. This process produces a map of the correlation between the point (and its surroundings) in the initial image and the points within that corresponding neighborhood in the subsequent image; the location of the maximum correlation represents the likely location of the initial point (and its surrounding template) within the subsequent image. In this study, NCC enabled semi-automated AVJ tracking that reduced the long postprocessing time previously required with manual AVJ tracking [19]. In addition, the use of reproducibility analyses here showed that the use of NCC limited variability in calculated MA sweep volume measurements between users.

Our results showed that patients with TTE-based diastolic dysfunction had lower normalized peak and average MA sweep rates in early diastole, resulting in the reversal of $\mathrm{PSR}_{E} / \mathrm{PSR}_{\mathrm{A}}$ ratios. Likewise, prior echocardiographic studies have reported the analogous phenomenon of TDI $\mathrm{e}^{\prime} / \mathrm{a}^{\prime}$ MA velocity reversal in diastolic dysfunction [21,29]. The present study also showed a relative increase in peak MA sweep rate in atrial systole in these patients. This can potentially be explained by the effect of LA dilatation associated with diastolic dysfunction, which may have led to compensatory enhancement of LA contraction, due to activation of the Frank-Starling mechanism [21]. Based on our data, LA dilatation was significantly associated with both CMR-based and TTE-based diastolic dysfunction compared to their respective controls. In addition, ROC analysis showed that the combined use of $\mathrm{PSR}_{\mathrm{E}}$ and $\mathrm{PSR}_{E} / \mathrm{PSR}_{\mathrm{A}}$ improved performance in identifying patients with TTE-based diastolic dysfunction. The ability to characterize atrial systole with our methods is an advantage compared to techniques such as MR tagging, which cannot reliably produce late diastolic strain rates because of fading signal intensities due to $\mathrm{T} 1$ relaxation [30].

MA sweep mechanics were reported here in terms of "percentage sweep volume recovery". This index showed that patients with normal diastolic function recovered nearly $70 \%$ of the end-systolic sweep volume by the end of early diastole, or $10.6 \mathrm{~mL}$ in absolute volume, but patients with diastolic dysfunction only recovered $54 \%$, or $6.8 \mathrm{~mL}$. These findings were in rough overall agreement with a prior study that reported an average MA excursion volume of $6 \mathrm{~mL}$ in nine healthy subjects [17]. However, this prior study involved subjects with a considerably lower average stroke volume $(52 \mathrm{~mL}$ vs. $93 \mathrm{~mL}$ ), which may explain the moderate difference in results. Overall, the CMR parameters discussed here were all consistent in demonstrating blunted early-diastolic MA kinetics in patients with abnormal TDI velocities.

We also investigated the time intervals of the MA sweep volume. The results showed that patients with TTE-based diastolic dysfunction had significantly longer

Table 3 Classification performance of CMR diastolic parameters

\begin{tabular}{|c|c|c|c|c|c|c|c|}
\hline & AUC & Test + Criterion & Sensitivity & Specificity & PPV & NPV & Accuracy \\
\hline $\mathrm{PSR}_{\mathrm{E}}$ & 0.88 & $<6.65$ & $89 \%$ & $74 \%$ & $77 \%$ & $87 \%$ & $81 \%$ \\
\hline Percentage sweep volume recovery, early diastole & 0.82 & $<57.90$ & $65 \%$ & $87 \%$ & $83 \%$ & $71 \%$ & $76 \%$ \\
\hline Normalized average sweep rate, early diastole & 0.93 & $<3.12$ & $85 \%$ & $87 \%$ & $87 \%$ & $86 \%$ & $86 \%$ \\
\hline $\mathrm{PSR}_{\mathrm{E}} / \mathrm{PSR}_{\mathrm{A}}$ & 0.88 & $<1.17$ & $84 \%$ & $81 \%$ & $81 \%$ & $83 \%$ & $82 \%$ \\
\hline $\mathrm{DT}_{\mathrm{sv}}$ & 0.83 & $>100.89$ & $82 \%$ & $74 \%$ & $76 \%$ & $81 \%$ & $78 \%$ \\
\hline DSVRT $_{50}$ & 0.79 & $>19.45$ & $63 \%$ & $84 \%$ & $80 \%$ & $69 \%$ & $73 \%$ \\
\hline
\end{tabular}

Classification performance results based on receiver operating characteristic (ROC) analysis. Threshold based on value yielding the greatest classification accuracy. $\mathrm{PSR}_{\mathrm{E}}=$ normalized peak sweep rate in early diastole; $\mathrm{PSR}_{\mathrm{A}}=$ normalized peak sweep rate in atrial systole; $\mathrm{DT}_{\mathrm{SV}}=\mathrm{sweep}$ volume deceleration time; $\mathrm{DSVRT}_{50}=50 \%$ diastolic sweep volume recovery time; $\mathrm{AUC}=$ area under the curve; $\mathrm{PPV}=$ positive predictive value; $\mathrm{NPV}=$ negative predictive value. 
Table 4 Multivariate analysis to predict TTE-based diastolic dysfunction

\begin{tabular}{|c|c|c|c|c|}
\hline & Odds Ratio & $95 \% \mathrm{Cl}$ & Coefficient (B) & $\mathbf{P}$ \\
\hline \multicolumn{5}{|l|}{ Model for PSR $_{E}$} \\
\hline Age & 1.07 & $1.02-1.12$ & 0.06 & $0.009^{*}$ \\
\hline HTN & 1.06 & $0.34-3.32$ & 0.06 & 0.919 \\
\hline LVH & 9.42 & $2.15-41.2$ & 2.24 & $0.003^{*}$ \\
\hline CAD & 1.22 & $0.35-4.21$ & 0.20 & 0.758 \\
\hline$P S R_{E}$ & 0.54 & $0.34-0.85$ & -0.62 & $0.007^{*}$ \\
\hline \multicolumn{5}{|l|}{ Model for $\mathrm{PSR}_{\mathrm{E}} / \mathrm{PSR}_{\mathrm{A}}$} \\
\hline Age & 1.07 & $1.02-1.13$ & 0.07 & $0.005^{*}$ \\
\hline HTN & 0.96 & $0.31-2.94$ & -0.04 & 0.944 \\
\hline LVH & 9.24 & $2.00-42.6$ & 2.22 & $0.004^{*}$ \\
\hline CAD & 0.93 & $0.27-3.24$ & -0.07 & 0.913 \\
\hline $\mathrm{PSR}_{\mathrm{E}} / \mathrm{PSR}_{\mathrm{A}}$ (per 0.1 increment) & 0.86 & $0.76-0.97$ & -0.15 & $0.016^{*}$ \\
\hline \multicolumn{5}{|l|}{ Model for $\mathrm{DT}_{\mathrm{SV}}$} \\
\hline Age & 1.10 & $1.05-1.15$ & 0.09 & $<0.001 *$ \\
\hline HTN & 1.05 & $0.35-3.18$ & 0.05 & 0.927 \\
\hline LVH & 10.45 & $2.44-44.8$ & 2.35 & $0.002^{*}$ \\
\hline CAD & 1.18 & $0.35-4.02$ & 0.17 & 0.791 \\
\hline $\mathrm{DT}_{\mathrm{SV}}$ (per 10 ms increment) & 1.12 & $0.99-1.28$ & 0.12 & $0.077 t$ \\
\hline \multicolumn{5}{|l|}{ Model for DSVRT $_{50}$} \\
\hline Age & 1.08 & $1.04-1.13$ & 0.08 & $<0.001^{*}$ \\
\hline HTN & 1.23 & $0.39-3.87$ & 0.20 & 0.726 \\
\hline LVH & 12.18 & $3.12-47.6$ & 2.50 & $<0.001 *$ \\
\hline CAD & 1.04 & $0.29-3.77$ & 0.04 & 0.950 \\
\hline DSVRT $_{50}$ & 1.14 & $1.03-1.26$ & 0.13 & $0.013^{*}$ \\
\hline
\end{tabular}

Results based on multivariate logistic regression analysis.

$\mathrm{Cl}=$ confidence interval.

$* P<0.05,+P<0.10$.

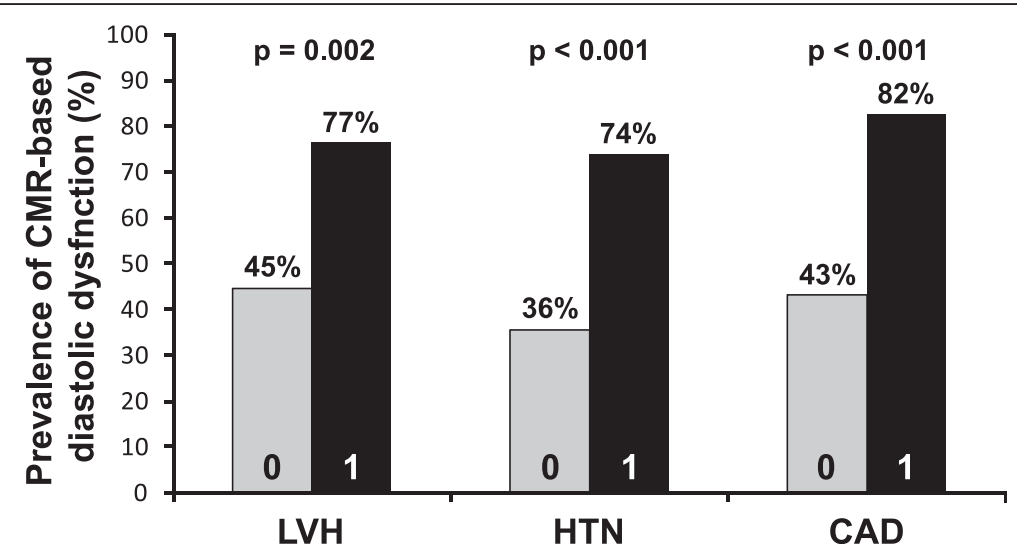

Figure 6 Prevalence of CMR-based diastolic dysfunction based on clinical status. A predictive model was constructed with multivariate logistic regression, using PSRE, PSRE/PSR $R_{A}$ DT $T_{S V}$, and DSVRT 50 as covariates to diagnose CMR-based diastolic dysfunction in patients with (1) and without (0) left ventricular hypertrophy (LVH), hypertension (HTN) and coronary artery disease (CAD). A higher prevalence of CMR-based diastolic dysfunction was observed in these clinical subgroups. 
Table 5 Reproducibility of CMR sweep volume indices

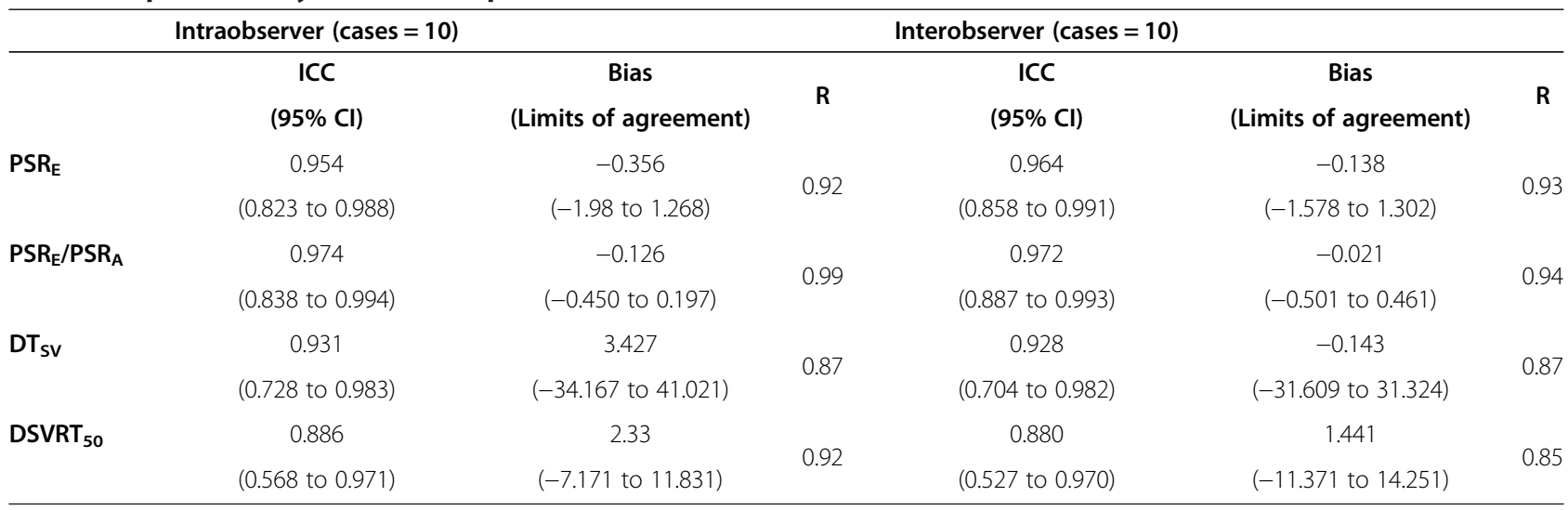

$\mathrm{ICC}=$ Intraclass correlation coefficient with $95 \%$ confidence interval $(\mathrm{Cl})$; bias and limits of agreement generated by Bland-Altman analysis; R correlation coefficient determined by Pearson's $r$ test.

$\mathrm{DT}_{\mathrm{SV}}(144 \pm 55 \mathrm{~ms})$ compared to the normal diastolic function group $(96 \pm 37 \mathrm{~ms})$. $\mathrm{AT}_{\mathrm{SV}}$ was slightly longer in the diastolic dysfunction group, but the difference was not significant. Both findings were consistent with a published TDI study that examined acceleration and deceleration times pertaining to MA velocities (normal DT $=84 \mathrm{~ms}$, diastolic dysfunction DT $=156$ to $168 \mathrm{~ms}$ ) [31].

DSVRT $_{50}$ was an additional CMR diastolic function parameter, which measured the time needed for the MA to recover $50 \%$ of its end systolic sweep volume, adjusted for R-R interval. On average, longer $\mathrm{DSVRT}_{50}$ times were observed in patients with TTE-based diastolic dysfunction. This parameter was analogous to an index used in a prior study named "diastolic volume recovery" [12], which accounted for both heart rate and volume status when assessing diastolic dysfunction in CMR LV volumetric filling. Lastly, it is important to note that $\mathrm{PSR}_{\mathrm{E}}, \mathrm{PSR}_{\mathrm{E}} / \mathrm{PSR}_{\mathrm{A}}$, and $\operatorname{DSVRT}_{50}$ each independently predicted TTE-based diastolic dysfunction after controlling for age, LVH, HTN, and CAD.

Clinically, the study demonstrated that patients with HTN, LVH, and CAD had increased prevalence of CMRbased diastolic dysfunction. Among the LVH subgroup, a large percentage (20/23 patients) was also diagnosed with hypertrophic cardiomyopathy. Similar results regarding these clinical subgroups have been reported in the past [27]. In general, HTN, LVH, and CAD are all well-known causes of diastolic dysfunction, and often co-exist in patients with cardiovascular disease. These conditions have been implicated in the disruption of myocyte calcium reuptake that governs the active phase of relaxation, as well as mechanisms that increase collagen synthesis and the ventricular stiffness that governs the passive phase of relaxation [32,33]. Because previous echocardiography studies have shown that diastolic dysfunction often develops before any clinically apparent changes of cardiac function in predisposed individuals
[34], such patients undergoing routine CMR may benefit from additional assessment of their diastolic function using the proposed method. In particular, the CMR-derived diastolic function variables may contribute additional value to the conventional CMR characterization of tissue morphology and contrast agent delayed enhancement.

The MA shape was reconstructed in 3D with six imaged AVJ points, derived from three standard LV long-axis planes situated approximately 60 degrees apart in space. By accounting for MA 3D motion in its entirety, in both transverse and longitudinal directions, the proposed method was hypothesized to be a better representation of global diastolic function than TDI, which only measures longitudinal motion. Typically in TDI, longitudinal velocities are measured regionally at the septal and lateral sides of the MA. However, prior studies have reported significant regional variability in LV myocardial velocities [35]. Specifically, early TDI velocities in the lateral, inferior, and posterior locations were often greater than the septal and anterior segments. As a result, varying regional wall motion abnormalities were shown to undermine the correlation between TDI velocities and invasively acquired LV end-diastolic pressure and tau [36]. In current practice, the average of TDI velocities is often used to better characterize global LV diastolic function [6]. In this study, CMR PSR $/ \mathrm{PSR}_{\mathrm{A}}$ correlated better with the average TDI $\mathrm{e}^{\prime} / \mathrm{a}^{\prime}$, compared to single-site measurements, thereby supporting the hypothesis that the proposed method inherently better characterizes global LV function. It is interesting to note that CMR $\mathrm{PSR}_{\mathrm{E}} / \mathrm{PSR}_{\mathrm{A}}$ correlated better with lateral TDI $e^{\prime} / a^{\prime}$ than it did with septal $e^{\prime} / a^{\prime}$. This finding is consistent with studies showing that, among single site measurements, lateral TDI velocities have the best correlations with global LV filling pressures and invasive indices of LV compliance [37].

The CMR-derived MA sweep volume was found here to effectively characterize global diastolic function. Prior 
echocardiography studies have demonstrated that MA excursion volume plays an important role in LV function $[17,38]$. In addition, reports have long recognized the relationship between LV long-axis function and MA displacement [39]. For example, the longitudinal MA excursion in diastole envelops and effectively transfers blood from the atrium to the ventricle, separately from the flow of blood across the location of the MA. This can occur even while the blood remains relatively stationary in relation to the apex. Similarly, in atrial systole, the MA is pulled away from the apex by the atrial pectinate muscles, "over" the blood, to facilitate further ventricular filling (and also increasing the ventricular pre-stretch, thus augmenting contractility). As a result, MA excursion is a central component of diastole, along with the transmitral pressure gradient.

\section{Limitations}

There were some limitations in our study design. First, this was a retrospective investigation and most patients did not have their imaging studies performed on the same day. Although the subjects were in stable clinical condition between studies, changes in cardiac function between studies were possible. This may explain some discrepancy in diastolic function as identified by CMR and TTE. Other potential reasons for discrepancy include differences in temporal resolution between the methods, as well as sampling location, where regional dysfunction identified by TDI may not correspond with global dysfunction as assessed by MA sweep volume [12], or vice versa. Second, the study used TTE as the standard reference for diastolic function instead of invasive hemodynamic data. However, routine use of invasive studies is not practical. Furthermore, despite various limitations of TTE [5], it remains the generally accepted non-invasive reference method for diastolic function assessment. Third, the study lacked follow-up data that might indicate the prognostic significance of the results of the proposed method.

There are also several limitations of the proposed technique. First, the three long-axis images used to reconstruct the MA were acquired at similar, but different, points in time, e.g., with potential differences in respiratory excursion. Second, the accuracy of NCC feature-tracking can be affected by image scaling, rotation, and distortion [23]; as with other feature-tracking approaches, it is dependent on there being some suitable distinctive image structure around the point being tracked. In conventional long-axis cine-CMR images, significant scaling or rotation were rarely seen in AVJ motion. However, distortion of the AVJ was relatively common during rapid motion, due to resulting blurring artifacts, which necessitated manual correction of the AVJ tracking results in a minority of cases. Lastly, when using routinely acquired long-axis images, only six points could be used to reconstruct the 3D MA. However, a prior animal study was able to effectively characterize MA dynamics by implanting only eight equally spaced radiopaque markers on the MA [38].

Future studies involving a larger cohort with assessment of the prognostic utility of the information may offer further evaluation of the potential clinical utility of CMR-derived 3D MA sweep volume as a technique for evaluation of diastolic dysfunction. A larger population could also allow stratification of the different diastolic dysfunction stages. In addition, there may be particular value in applying the technique in evaluating specific patient groups with potential ventricular dysfunction, such as athletes, patients with hypertrophic, dilated, or restrictive cardiomyopathies, or patients undergoing anthracycline therapy.

\section{Conclusion}

3D MA sweep volumes generated by semi-automated AVJ tracking in routinely acquired CMR images demonstrated diastolic parameters that were effective in identifying patients with TTE-based diastolic dysfunction. These parameters also correlated well with TDI MA velocities, and may serve as a useful tool for characterizing patients with diastolic dysfunction.

\section{Abbreviations \\ CMR: Cardiovascular magnetic resonance; MA: Mitral annulus; TDI: Tissue Doppler imaging; TTE: Transthoracic echocardiography; TMF: Transmitral flow: AVJ: Atrioventricular junction; PSR: Peak sweep rate; $\mathrm{DT}_{\text {sV: }}$. Deceleration time of sweep volume; $\mathrm{AT}_{\mathrm{sv}}$ : Acceleration time of sweep volume; DSVRT $_{50}$ : $50 \%$ diastolic sweep volume recovery time; ESSV: End systolic sweep volume; AUC: Area-under-the-curve; ROC: Receiver-operator characteristic; ICC: Intraclass correlation coefficients.}

\section{Competing interests}

The authors declare that they have no competing interests.

\section{Authors' contributions}

WW: conception and design, development and implementation of algorithm and software, acquisition, analysis and interpretation of data, statistical analysis, drafting of manuscript; JC: acquisition, analysis and interpretation of data, statistical analysis, critical revision of manuscript; SC: acquisition, analysis and interpretation of data, development of original software, critical revision of manuscript; SB: development of original software, critical revision of manuscript; LA: conception and design, acquisition, analysis and interpretation of data, critical revision of manuscript; All authors read and approved the final manuscript.

\section{Acknowledgements}

This study was supported by the National Institutes of Health grant R21-HL108218.

We thank James S. Babb, PhD (Associate Professor, New York University School of Medicine), who provided statistical advice.

Received: 12 March 2014 Accepted: 26 August 2014

Published online: 20 September 2014

\section{References}

1. Hogg K, Swedberg K, McMurray J. Heart failure with preserved left ventricular systolic function; epidemiology, clinical characteristics, and prognosis. J Am Coll Cardiol. 2004; 43:317-27. 
2. Meta-analysis Global Group in Chronic Heart Failure. The survival of patients with heart failure with preserved or reduced left ventricular ejection fraction: an individual patient data meta-analysis. Eur Heart $\mathrm{J}$. 2012; 33:1750-57.

3. Paulus WJ, Tschöpe C, Sanderson JE, Rusconi C, Flachskampf FA, Rademakers FE, Marino P, Smiseth OA, De Keulenaer G, Leite-Moreira AF. How to diagnose diastolic heart failure: a consensus statement on the diagnosis of heart failure with normal left ventricular ejection fraction by the Heart Failure and Echocardiography Associations of the European Society of Cardiology. Eur Heart J. 2007; 28:2539-50.

4. Dougherty AH, Naccarelli GV, Gray EL, Hicks CH, Goldstein RA. Congestive heart failure with normal systolic function. Am J Cardiol. 1984; 54:778-82.

5. Fyrenius A, Wigström L, Bolger AF, Ebbers T, Öhman KP, Karlsson M, Wranne $B$, Engvall J. Pitfalls in Doppler evaluation of diastolic function: insights from 3-dimensional magnetic resonance imaging. J Am Soc Echocardiogr. 1999; 12:817-26.

6. Rivas-Gotz C, Manolios M, Thohan V, Nagueh SF. Impact of left ventricular ejection fraction on estimation of left ventricular filling pressures using tissue Doppler and flow propagation velocity. Am J Cardiol. 2003; 91:780-84.

7. Rivas-Gotz C, Khoury DS, Manolios M, Rao L, Kopelen HA, Nagueh SF. Time interval between onset of mitral inflow and onset of early diastolic velocity by tissue Doppler: a novel index of left ventricular relaxation: experimental studies and clinical application. J Am Coll Cardiol. 2003; 42:1463-70.

8. Boogers MJ, Van Werkhoven JM, Schuijf JD, Delgado V, El-Naggar HM, Boersma E, Nucifora G, van der Geest RJ, Paelinck BP, Kroft L. Feasibility of Diastolic Function Assessment With Cardiac CT: Feasibility Study in Comparison With Tissue Doppler Imaging. JACC Cardiovasc Imaging. 2011; 4:246-56.

9. Caudron J, Fares J, Bauer F, Dacher J-N. Evaluation of left ventricular diastolic function with cardiac MR imaging. Radiographics. 2011; 31:239-59.

10. Rathi VK, Doyle M, Yamrozik J, Williams RB, Caruppannan K, Truman C, Vido D, Biederman RW. Routine evaluation of left ventricular diastolic function by cardiovascular magnetic resonance: A practical approach. J Cardiovasc Magn Reson. 2008; 10:A212.

11. Paelinck BP, De Roos A, Bax JJ, Bosmans JM, van Der Geest RJ, Dhondt D, Parizel PM, Vrints $C J$, Lamb HJ. Feasibility of tissue magnetic resonance imaging: a pilot study in comparison with tissue Doppler imaging and invasive measurement. J Am Coll Cardiol. 2005; 45:1109-16.

12. Kawaji K, Codella NC, Prince MR, Chu CW, Shakoor A, LaBounty TM, Min JK, Swaminathan RV, Devereux RB, Wang Y, Weinsaft JW. Automated segmentation of routine clinical cardiac magnetic resonance imaging for assessment of left ventricular diastolic dysfunction. Circ Cardiovasc Imaging. 2009; 2:476-84.

13. Mendoza DD, Codella NC, Wang Y, Prince MR, Sethi S, Manoushagian S., Kawaji K, Min JK, LaBounty TM, Devereux RB. Impact of diastolic dysfunction severity on global left ventricular volumetric filling-assessment by automated segmentation of routine cine cardiovascular magnetic resonance. J Cardiovasc Magn Reson. 2010; 12:46.

14. Garot J. The study of diastole by tagged MRI: are we nearly there yet? Eur Heart J. 2004; 25:1376-77.

15. Fernández-Pérez G, Duarte R, Corral De la Calle M, Calatayud J, Sánchez González J. Analysis of left ventricular diastolic function using magnetic resonance imaging. Radiología. 2012; 54:295-305.

16. Nammas W, El-Okda E. Atrioventricular plane displacement: does it predict in-hospital outcome after acute myocardial infarction? Eur Rev Med Pharmacol Sci. 2012; 16:16-21.

17. Carlhäll C, Wigström L, Heiberg E, Karlsson M, Bolger A, Nylander E. Contribution of mitral annular excursion and shape dynamics to total left ventricular volume change. Am J Physiol Heart Circ Physiol. 2004; 287:H1836-41

18. Maffessanti F, Gripari P, Pontone G, Andreini D, Bertella E, Mushtaq S, Tamborini G, Fusini L, Pepi M, Caiani EG. Three-dimensional dynamic assessment of tricuspid and mitral annuli using cardiovascular magnetic resonance. Eur Heart J Cardiovasc Imaging. 2013; 14:986-95.

19. Saba SG, Chung S, Bhagavatula S, Donnino R, Srichai MB, Saric M, Katz SD, Axel L. A novel and practical cardiovascular magnetic resonance method to quantify mitral annular excursion and recoil applied to hypertrophic cardiomyopathy. J Cardiovasc Magn Reson. 2014; 16:1-9.

20. Naqueh SF, Appleton CP, Gillebert TC, Marino PN, Oh JK, Smiseth OA, Waggoner AD, Flachskampf FA, Pellikka PA, Evangelisa A.

Recommendations for the evaluation of left ventricular diastolic function by echocardiography. Eur J Echocardiogr. 2009; 10:165-93.
21. Morris DA, Gailani M, Vaz Pérez A, Blaschke F, Dietz R, Haverkamp W, Özcelik C. Left atrial systolic and diastolic dysfunction in heart failure with normal left ventricular ejection fraction. J Am Soc Echocardiogr. 2011; 24:651-62.

22. Lang RM, Bierig M, Devereux RB, Flachskampf FA, Foster E, Pellikka PA, Picard MH, Roman MJ, Seward J, Shanewise J. Recommendations for chamber quantification. Eur J Echocardiogr. 2006; 7:79-108.

23. Lewis J. Fast normalized cross-correlation. Vision Interface. 1995; 10:120-23.

24. Hii A, Hann CE, Chase JG, Van Houten EE. Fast normalized cross correlation for motion tracking using basis functions. Comput Methods Programs Biomed. 2006; 82:144-56.

25. Levine RA, Handschumacher MD, Sanfilippo A, Hagege A, Harrigan P, Marshall J, Weyman A. Three-dimensional echocardiographic reconstruction of the mitral valve, with implications for the diagnosis of mitral valve prolapse. Circulation. 1989; 80:589-98.

26. Ennis DB, Rudd-Barnard GR, Li B, Fonseca CG, Young AA, Cowan BR, Stewart RA. Changes in mitral annular geometry and dynamics with $\beta$-blockade in patients with degenerative mitral valve disease. Circ Cardiovasc Imaging. 2010; 3:687-93.

27. Fischer M, Baessler A, Hense $H$, Hengstenberg C, Muscholl M, Holmer S, Döring A, Broeckel U, Riegger G, Schunkert H. Prevalence of left ventricular diastolic dysfunction in the community. Results from a Doppler echocardiographic-based survey of a population sample. Eur Heart J. 2003; 24:320-28.

28. Zile MR, Brutsaert DL. New concepts in diastolic dysfunction and diastolic heart failure: Part I diagnosis, prognosis, and measurements of diastolic function. Circulation. 2002; 105:1387-93.

29. Sohn DW, Chai IH, Lee DJ, Kim HC, Kim HS, Oh BH, Lee MM, Park YB, Choi YS, Seo JD. Assessment of mitral annulus velocity by Doppler tissue imaging in the evaluation of left ventricular diastolic function. J Am Coll Cardiol. 1997; 30:474-80.

30. Edvardsen T, Rosen BD, Pan L, Jerosch-Herold M, Lai S, Hundley WG, Sinha S, Kronmal RA, Bluemke DA, Lima JA. Regional diastolic dysfunction in individuals with left ventricular hypertrophy measured by tagged magnetic resonance imaging - the Multi-Ethnic Study of Atherosclerosis (MESA). Am Heart J. 2006; 151:109-14.

31. Nagueh SF, Middleton KJ, Kopelen HA, Zoghbi WA, Quiñones MA. Doppler tissue imaging: a noninvasive technique for evaluation of left ventricular relaxation and estimation of filling pressures. J Am Coll Cardiol. 1997; 30:1527-33.

32. Choudhury L, Gheorghiade M, Bonow RO. Coronary artery disease in patients with heart failure and preserved systolic function. Am J Cardiol. 2002: 89:719-22.

33. Verma A, Solomon SD. Diastolic dysfunction as a link between hypertension and heart failure. Med Clin North Am. 2009; 93:647-64.

34. Aeschbacher BC, Hutter D, Fuhrer J, Weidmann P, Delacrétaz E, Allemann Y. Diastolic dysfunction precedes myocardial hypertrophy in the development of hypertension. Am J Hypertens. 2001; 14:106-13.

35. Galiuto L, Ignone G, DeMaria AN. Contraction and relaxation velocities of the normal left ventricle using pulsed-wave tissue Doppler echocardiography. Am J Cardiol. 1998; 81:609-14.

36. Fujii T, Yoshioka K, Nakano M, Nakazawa G, Amino M, Masuda N, Shinozaki N, Kanda S, Ogata N, Deguchi Y. Regional wall motion abnormality at the lateral wall disturbs correlations between tissue Doppler E/e' ratios and left ventricular diastolic performance parameters measured by invasive methods. J Echocardiogr. 2013; 11:138-46.

37. Kasner M, Westermann D, Steendijk P, Gaub R, Wilkenshoff U, Weitmann K Hoffmann W, Poller W, Schultheiss H-P, Pauschinger M. Utility of Doppler Echocardiography and Tissue Doppler Imaging in the Estimation of Diastolic Function in Heart Failure With Normal Ejection Fraction A Comparative Doppler-Conductance Catheterization Study. Circulation. 2007; 116:637-47.

38. Carlhäll C, Kindberg K, Wigström L, Daughters GT, Miller DC, Karlsson M, Ingels NB. Contribution of mitral annular dynamics to LV diastolic filling with alteration in preload and inotropic state. Am J Physiol Heart Circ Physiol. 2007; 293:H1473-79.

39. Henein MY, Gibson DG. Normal long axis function. Heart. 1999; 81:111-13.

\section{doi:10.1186/s12968-014-0071-3}

Cite this article as: Wu et al.: Evaluation of diastolic function by three-dimensional volume tracking of the mitral annulus with cardiovascular magnetic resonance: comparison with tissue Doppler imaging. Journal of Cardiovascular Magnetic Resonance 2014 16:71. 